\section{Zolpidem-induced somnambulism in an elderly man}

Dear Editor - We describe a case demonstrating an association between zolpidem and somnambulism in an elderly man. The implications of this are discussed. Zolpidem is an imidazopyridine non-benzodiazepine short acting hypnotic that acts on benzodiazepine receptor sites in the cerebellum and cerebral cortex. ${ }^{1}$ Usual therapeutic dose is $5-10 \mathrm{mg}$, and time until onset of action is 7-27 minutes. Because of its rapid onset and short duration of action, it is commonly used as a hypnotic among people of all ages, including older subjects. We describe a case of somnambulism associated with its use.

\section{Case report}

Mr DB was a 79 year old gentleman who was referred to the Old Age Psychiatry service because of three episodes of unusual and uncharacteristic behaviour, described as hallucinations. He was resident in sheltered accommodation for a number of months as he had lived alone and his physical health was declining. The episodes consisted of him getting up during the night and walking around the home. On one occasion he dressed himself at $4 \mathrm{am}$, on another he was incontinent of urine, and on the third he carried objects with him, including a mobile phone and a hot water bottle. He spoke to staff but the content made no sense. He had no recall of these incidents the following days and was upset to hear he had behaved in such a way.

$\mathrm{He}$ had no past psychiatric history. He had a medical history comprising heart failure, for which he attended a heart failure clinic, CVA five years previously with a good recovery, hypertension, pulmonary fibrosis and a UTI three months previously requiring hospital inpatient treatment and during which he had an episode of delirium and visual hallucinations. On mental state examination, other than some anxiety about the above events, there was no evidence of psychiatric illness. He did not have an affective disorder or psychosis and cognitive function was intact.

His medication included Nuseals Aspirin, furosemide, bisoprolol, escitalopram $5 \mathrm{mg} \mathrm{OD}$, atorvastatin $10 \mathrm{mg}$ nocte, valsartan $8 \mathrm{mg} O D$, tamsulosin and zolpidem $10 \mathrm{mg}$ nocte. On review of his medication, zolpidem had been given at an increased dose of $20 \mathrm{mg}$ nocte for two to three weeks prior to the nocturnal wandering. He had previously been on $10 \mathrm{mg}$ nocte. This was reduced to $10 \mathrm{mg}$ after the third episode and no further episodes occurred. No other alterations had been made to his medications in the previous month.

\section{Discussion}

Several case reports of unusual side-effects of zolpidem have been described, including somnambulism, amnesia, visual hallucinations, sleep-related eating disorder and compulsive behaviour, such as repetitive cleaning, shopping and eating. . $^{2-5}$

Zolpidem-associated parasomnia has been described in younger adults. In one case a 51 year old woman developed somnambulism and nocturnal eating after taking zolpidem $10 \mathrm{mg}$ nocte for two months. ${ }^{6} \mathrm{~A} 19$ year old man with a diagnosis of DSM-IV schizoaffective disorder and impulse control disorder experienced somnambulism and incoherent speech one to two days after commencing zolpidem for medication associated insomnia. ${ }^{7} \mathrm{~A}$ man in his mid 50 s with a history of traumatic brain injury developed somnambulism with associated amnesia and urinary incontinence after being prescribed $10 \mathrm{mg}$ zolpidem following a right hip hemiarthroplasty. ${ }^{8}$ In all these cases, symptoms ceased on discontinuation of zolpidem.

Somnambulism associated with zolpidem may be diagnosed easily if there is a clear cut temporal relationship between the commencement of the drug and onset of symptoms. However it may not be so obvious in all cases, as in our patient where an increase in dose had occurred. Additionally, in an elderly person other potential causes for the behaviour may be suspected - such as delirium, which our patient had recently had, or nocturnal confusion in association with an underlying or evolving dementia. Awareness of the possibility of drug-induced somnambulism will enable rapid and effective action to be taken. Failure to consider this may lead to ongoing symptoms, anxiety for the patient and family and inappropriate medical investigations.

We feel it is important to highlight this unusual side-effect of zolpidem and recommend that clinicians review hypnotic use and dosage when patients present with new onset parasomniac activities.

Siobhan Madden, Registrar, Dept of Old Age Psychiatry, Carew House, St Vincent's University Hospital, Elm Park Dublin 4, Ireland.

Joseph Yasbeck, Medical Officer, Leopardstown Park Hospital, Foxrock Dublin 18, Ireland. *Aideen Freyne, Consultant Psychiatrist, Dept of O!d Age Psychiatry, Carew House, St Vincent's University Hospital, Elm Park Dublin 4, Ireland.

*Correspondence

\footnotetext{
References

1. Hoehns JD, Perry PJ. Zolpidem: a nonbenzodiazepine hypnotic for treatment of insomnia. Clin Pharm 1993; 12: 814-28.

2. Tsai JH, Yang P, Chen CC et al. Zolpidem-induced amnesia and somnambulism: rare occurrences? Eur Neuropsychopharmacol 2008;

3. Tsai MJ, Huang YB, Wu PC. A novel clinical pattern of visual hallucination after zolpidem use. J Toxicol Clin Toxicol 2003; 41: 869-72.

4. Najjar M. Zolpidem and amnestic sleep related eating disorder. J Clin Sleep Med 2007; $15: 637-8$.

5. Tsai MJ, Tsai YH, Huang YB. Compulsive activity and anterograde amnesia after zolpidem use. Clin Toxicol 2007; 45: 179-81.

6. Sansone RA, Sanson LA. Zolpidem, somnambulism and nocturnal eating. Gen Hosp Psychiatry 2008; 30: 90-1.

7. Sharma A, Dewan VK. A case report of zolpidem induced somnambulism. Prim Care Companion J Clin Psychiatry 2005; 7: 74.

8. Yang W, Dollear M, Muthukrishnan SR. One rare side effect of zolpidem sleepwalking: a case report. Arch Phys Med Rehabil 2005; 86: 1265-6.
} 
